\title{
WEAR OF ELASTIC FOUNDATION WITH COATING BY SYSTEM OF RIGID PUNCHES
}

\begin{abstract}
K. Kazakov *
Abstract: This article is devoted to the study of the wear problem of elastic layer with nonuniform coating by the system of plane rigid punches. It is assumed that punches has different lengths. A mathematical model of the problem is obtained. It is a system of mixed integral equations (with Volterra and Fredholm operators) with additional conditions. The analytical solution of the problem is presented. In the solution coating nonuniformity is represented by separate factor. It allows one to make calculations with high accuracy even if coating nonuniformity is described by rapidly changing function.
\end{abstract}

Keywords: Contact problem, Linear wear, Systems of integral equations, Projection method.

\section{Introduction}

In previous works some contact-wear problems for foundations with coatings were solved. Work Manzhirov and Kazakov (2017) was dedicated to the solving of the plane contact-wear problem for foundation with longitudially nonuniform coating and flat rigid punch. Paper Manzhirov and Kazakov (2018) considered an axisymmetric contact-wear problem for the case of complex shape of rigid punch. This paper provides solutions to the problem for the case when the foundation wears out simultaneously with several independent punches: different forces acting on the punches, punches has different lengths, distance between adjacent punches are not the same. The presented studies use some results from Kazakov et al (2017); Kazakov $(2018,2019)$.

\section{Statement of the contact-wear plane problem and mathematical model}

Elastic layer of arbitrary thickness $h_{\text {lower }}$ lies on an underlying undeformable basis without slipping (ideal contact). Another elastic layer of another thickness $h$ lies on the first layer. There is also ideal contact between the layers. The upper layer is softer than the bottom layer and it's elastic properties (Young modulus and Poisson's ratio) depends on longitudinal coordinate, i.e. $E=E(x), \nu=\nu(x)$. At the time $\tau_{0}$ several different punches begin to indent into described foundation along the axis $O z$. There is smooth contact between punches and upper layer along $O x$-axis. But the punches moves along the axis $O y$ perpendicular to the plane $O x z$ with average modulus of the punch velocity $V$. It is assumed that there is a friction between punches and upper layer along the axis $O y$ and it is characterized by coefficient $k_{\mathrm{w}}$. This coefficient depends, for example, on punch profiles along $O y$-axis. Further it is assumed that the contact regions are constant and bounded by segments $\bigcup_{i=1,2, \ldots, n}\left[a_{i}, b_{i}\right]$ ( $n$ is number of punches), the characteristic dimension $\bar{a}=\min _{i=1,2, \ldots, n} \bar{a}_{i}$ of the contact region is much greater than the upper layer thickness $h$ (upper layer is coating). Here $a_{i}$ and $b_{i}$ are left and right coordinates of $i$ th punch and $\bar{a}_{i}=b_{i}-a_{i}$ is width of this punch. The scheme of the problem is shown in the Figure 1.

Numerous experiments have shown that, in the case of linear wear, the wear velocity is proportional to the normal load and punch velocity and inversely proportional to the material hardness (see, for example,

Senior Researcher, Assoc. Prof. Kirill E. Kazakov, PhD.: Ishlinsky Institute for Problems in Mechanics RAS, Prospekt Vernadskogo 101-1, 119526, Moscow, Russia; Bauman Moscow State Technical University, 2-ya Baumanskaya ulitsa 5-1, 105005, Moscow, Russia; kazakov-ke@yandex.ru 


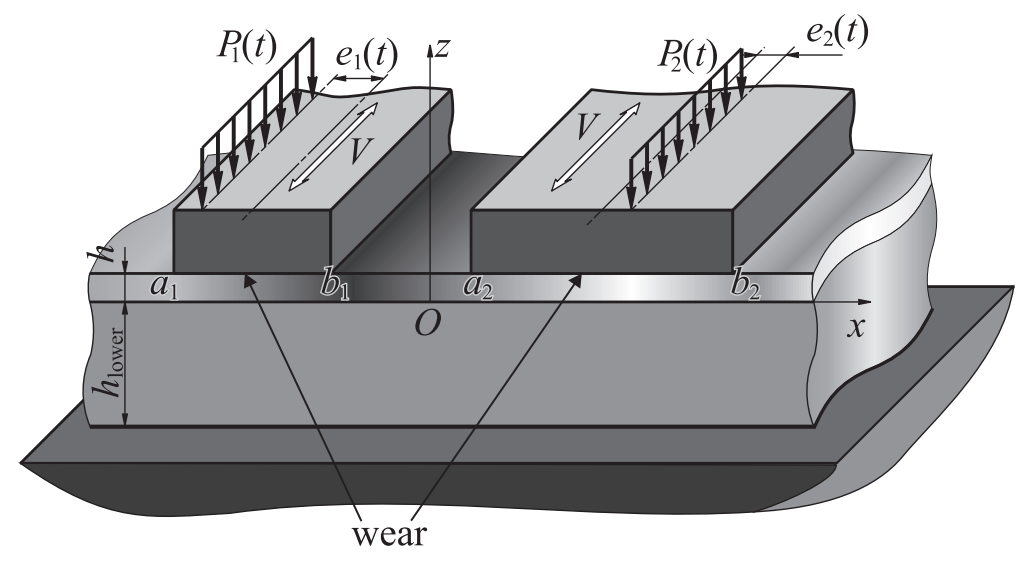

Fig. 1: Contact-wear problem for two punches

Pronikov (1957); Collins (1993); Soldatenkov (2010)). Then the vertical displacement of the top face of coating due to wear has the form

$$
u_{\mathrm{w}}(x, t)=-\frac{k_{\mathrm{w}} V}{H(x)} \int_{\tau_{0}}^{t} q(x, \tau) d \tau, \quad i=1,2,
$$

where $-q(x, t)$ is applied load, $H(x)$ is hardness of coating (upper layer). The vertical displacement of the coating top face due to applied load $-q(x, t)$ has a form (see, for example, Alexandrov and Kovalenko (1986))

$$
u_{\mathrm{q}}(x, t)=-\frac{q(x, t) h}{R(x)}-\frac{2\left(1-\nu_{\text {lower }}^{2}\right)}{\pi E_{\text {lower }}} \int_{-\infty}^{\infty} k_{\mathrm{pl}}\left(\frac{x-\xi}{h_{\text {lower }}}\right) q(\xi, t) d \xi
$$

Here $R(x)=E(x)[1-\nu(x)] /\left[1-\nu(x)-2 \nu^{2}(x)\right] ; \nu_{\text {lower }}$ and $E_{\text {lower }}$ are the Poisson's ratios and the Young modulus of the bottom layer; $k_{\mathrm{pl}}(s)$ is the plane contact problem kernel (see Vorovich et al (1974)). So total displacements under the punches equal to a sum of displacement due to wear (1) and displacement due to load (2). On the other hand total displacement of each punch equals $u_{i}(x, t)=-\delta_{i}(t)-\alpha_{i}(t)\left(x-\eta_{i}\right)$, where $\delta_{i}(t), \alpha_{i}(t)$, and $\eta_{i}=0.5\left(a_{i}+b_{i}\right)$ are $i$ th punch settlement, tilt angle, and midpoint. Hence main system of integral equations of the problem has a form $\left(x \in\left[a_{i}, b_{i}\right], t \geq \tau_{0}, i=1,2, \ldots, n\right)$

$$
\frac{k_{\mathrm{w}} V}{H(x)} \int_{\tau_{0}}^{t} q_{i}(x, \tau) d \tau+\frac{q_{i}(x, t) h}{R(x)}+\frac{2\left(1-\nu_{\text {lower }}^{2}\right)}{\pi E_{\text {lower }}} \sum_{j=1}^{n} \int_{a_{j}}^{b_{j}} k_{\mathrm{pl}}\left(\frac{x-\xi}{h_{\text {lower }}}\right) q_{j}(\xi, t) d \xi=\delta_{i}(t)+\alpha_{i}(t)\left(x-\eta_{i}\right),
$$

The resulting equation should be supplemented by equilibrium conditions of the punches:

$$
\int_{a_{i}}^{b_{i}} q_{i}(x, t) d x=P_{i}(t), \quad \int_{a_{i}}^{b_{i}}\left(x-\eta_{i}\right) q(x, t) d x=P_{i}(t) e_{i}(t), \quad t \geq \tau_{0}, \quad i=1,2, \ldots, n,
$$

where $P_{i}(t)$ are applied forces and $e_{i}(t)$ are eccentricities.

There will be cases when the hardness of the material is proportional to it's rigidity (see, for example, Archard (1953) and Schalliamach (1954)), i.e. $H(x)=k_{\mathrm{H}} R(x)$.

We use following change of variables in equations (3), (4):

$$
\begin{aligned}
& x^{*}=\frac{2\left(x-\eta_{i}\right)}{\bar{a}_{i}}, \quad \xi^{*}=\frac{2\left(\xi-\eta_{j}\right)}{\bar{a}_{j}}, \quad t^{*}=\frac{t}{\tau_{0}}, \quad \lambda=\frac{2 h_{\text {lower }}}{\bar{a}}, \quad \zeta^{i *}=\frac{\bar{a}_{i}}{\bar{a}}, \quad \eta^{i *}=\frac{2 \eta_{i}}{\bar{a}}, \\
& \delta^{i *}\left(t^{*}\right)=\frac{2 \delta_{i}(t)}{\bar{a}}, \quad \alpha^{i *}\left(t^{*}\right)=\zeta^{i *} \alpha_{i}(t), \quad V^{*}=\frac{k_{\mathrm{w}} V \tau_{0}}{k_{\mathrm{H}} h}, \quad m^{i *}\left(x^{*}\right)=\frac{k_{\mathrm{H}} h E_{\text {lower }}}{\bar{a}_{i} H(x)\left(1-\nu_{\text {lower }}^{2}\right)}, \\
& q^{i *}\left(x^{*}, t^{*}\right)=\frac{2 \zeta^{i *} q_{i}(x, t)\left(1-\nu_{\text {lower }}^{2}\right)}{E_{\text {lower }}}, \quad k^{i j}\left(x^{*}, \xi^{*}\right)=\frac{1}{\pi} k_{\mathrm{pl}}\left(\frac{\zeta^{i *} x^{*}+\eta^{i *}-\zeta^{j *} \xi^{*}-\eta^{j *}}{\lambda}\right) \\
& P^{i *}\left(t^{*}\right)=\frac{4 P_{i}(t)\left(1-\nu_{\text {lower }}^{2}\right)}{\bar{a} E_{\text {lower }}}, \quad M^{i *}\left(t^{*}\right)=\frac{8 P_{i}(t) e_{i}(t)\left(1-\nu_{\text {lower }}^{2}\right)}{\bar{a}_{i} \bar{a} E_{\text {lower }}}, \\
& x \in\left[a_{i}, b_{i}\right], \quad \xi \in\left[a_{j}, b_{j}\right], \quad t \geq \tau_{0}, \quad i, j=1,2, \ldots, n .
\end{aligned}
$$


Having omitted asterisks in the relations obtained, we arrive at the system integral equation and system of additional condition in the form

$$
\begin{aligned}
& m^{i}(x)\left[q^{i}(x, t)+V \int_{1}^{t} q^{i}(x, \tau) d \tau\right]+\sum_{j=1}^{n} \int_{-1}^{1} k^{i j}(x, \xi) q^{j}(\xi, t) d \xi=\delta^{i}(t)+\alpha^{i}(t) x, \\
& \int_{-1}^{1} q^{i}(x, t) d x=P^{i}(t), \quad \int_{-1}^{1} x q^{i}(x, t) d x=M^{i}(t), \quad x \in[-1,1], \quad t \geq 1, \quad i=1,2, \ldots, n,
\end{aligned}
$$

or in vector form

$$
\begin{aligned}
& \mathbf{D}(x) \cdot(\mathbf{I}-\mathbf{V}) \mathbf{q}(x, t)+\mathbf{F} \mathbf{q}(x, t)=\boldsymbol{\delta}(t)+\boldsymbol{\alpha}(t) x, \\
& \int_{-1}^{1} \mathbf{q}(x, t) d x=\mathbf{P}(t), \quad \int_{-1}^{1} x \mathbf{q}(x, t) d x=\mathbf{M}(t), \quad x \in[-1,1], \quad t \geq 1,
\end{aligned}
$$

where $\mathbf{D}(x)$ is diagonal matrix with elements $m^{i}(x)$ and

$$
\begin{aligned}
& \mathbf{q}(x, t)=\sum_{i=1}^{n} q^{i}(x, t) \mathbf{i}^{i}, \quad \boldsymbol{\delta}(t)=\sum_{i=1}^{n} \delta^{i}(t) \mathbf{i}^{i}, \quad \boldsymbol{\alpha}(t)=\sum_{i=1}^{n} \alpha^{i}(t) \mathbf{i}^{i}, \quad \mathbf{P}(t)=\sum_{i=1}^{n} P^{i}(t) \mathbf{i}^{i}, \quad \mathbf{M}(t)=\sum_{i=1}^{n} M^{i}(t) \mathbf{i}^{i}, \\
& \mathbf{k}(x, \xi)=\sum_{i, j=1}^{n} k^{i j}(x, \xi) \mathbf{i}^{i} \mathbf{i}^{j}, \quad \mathbf{F f}(x)=\int_{-1}^{1} \mathbf{k}(x, \xi) \cdot \mathbf{f}(\xi) d \xi, \quad \mathbf{V} f(t)=-V \int_{1}^{t} f(\tau) d \tau .
\end{aligned}
$$

Note that functions $m^{i}(x)$ in (6) (or matrix $\mathbf{D}(x)$ in (7)) connect with hardness of coating which can be described by a rapidly changing and discontinuous functions. So it is required to solve mixed operator equation (i.e. equations with two different integral operators, see Manzhirov (2016)) with several rapidly changing functions.

\section{Solution of the problem}

Note that there is exist fifteen versions of mathematical statements for the plane contact problem for a system of punches (because there are exist four different condition types on each punch and we can combine punches in groups with similar condition types). Solution constructing will be similar for all versions.

Operator equation (7) has a structure close to the modified operator equation (8) from Kazakov (2019): the difference is only in the position of Volterra operator. Additional conditions are the same. It can be shown that both the method and the process of constructing the solution are similar. So final solution for the case when all forces and moments are known has a form $(x \in[-1,1], t \geq 1, i=1,2, \ldots, n)$

$$
\begin{aligned}
& q^{i}(x, t)=\frac{1}{m^{i}(x)}\left[z_{0}^{i}(t) p_{0}^{i \circ}(x)+z_{1}^{i}(t) p_{1}^{i \circ}(x)+\sum_{k=2}^{\infty} z_{k}(t) \sum_{m=2}^{\infty} \psi_{k m}^{i} p_{m}^{i \circ}(x)\right], \\
& \alpha^{i}(t)=\sqrt{\frac{J_{0, i}}{J_{0, i} J_{2, i}-J_{1, i}^{2}}}\left\{z_{1}^{i}(t)+\int_{1}^{t} z_{1}^{i}(\tau) d \tau+\sum_{j=1}^{n}\left[\sum_{l=0}^{1} K_{0 l}^{i j} z_{l}^{j}(t)+\sum_{k=2}^{\infty}\left(\sum_{l=2}^{\infty} K_{0 l}^{i j} \psi_{k l}^{j}\right) z_{k}(t)\right]\right\}, \\
& \delta^{i}(t)=\frac{1}{\sqrt{J_{0, i}}}\left\{z_{0}^{i}(t)+\int_{1}^{t} z_{0}^{i}(\tau) d \tau+\sum_{j=1}^{n}\left[\sum_{l=0}^{1} K_{1 l}^{i j} z_{l}^{j}(t)+\sum_{k=2}^{\infty}\left(\sum_{l=2}^{\infty} K_{1 l}^{i j} \psi_{k l}^{j}\right) z_{k}(t)\right]\right\}-\frac{J_{1, i}}{J_{0, i}} \alpha^{i}(t),
\end{aligned}
$$

where $(i, j=1,2, \ldots, n, k=2,3,4, \ldots, m, l=0,1,2, \ldots)$

$$
\begin{aligned}
& z_{0}^{i}(t)=\frac{P^{i}(t)}{\sqrt{J_{0, i}}}, \quad z_{1}^{i}(t)=\frac{J_{0, i} P^{i}(t)+J_{1, i} M^{i}(t)}{\sqrt{J_{0, i}\left(J_{0, i} J_{2, i}-J_{1, i}^{2}\right)}}, \quad J_{l, i}=\int_{-1}^{1} \frac{\xi^{l}}{m^{i}(\xi)} d \xi \\
& z_{k}(t)=\frac{-\sigma_{k}(t)}{1+\gamma_{k}}+\frac{V}{\left(1+\gamma_{k}\right)^{2}} \int_{0}^{t} \sigma_{k}(\tau) \exp \left[-\frac{V(t-\tau)}{1+\gamma_{k}}\right] d \tau, \quad \sigma_{k}(t)=\sum_{l=1}^{2} \sum_{i, j=1}^{n} \sum_{m=2}^{\infty} K_{m l}^{i j} \psi_{k m}^{i} z_{l}^{j}(t), \\
& p_{l}^{i \circ}(x)=\frac{1}{\sqrt{d_{l-1, i} d_{l, i}}}\left|\begin{array}{cccc}
J_{0, i} & J_{1, i} & \cdots & J_{l, i} \\
J_{1, i} & J_{2, i} & \cdots & J_{l+1, i} \\
\vdots & \vdots & \ddots & \vdots \\
1 & x & \cdots & x^{l}
\end{array}\right|, \quad d_{-1, i}=1, \quad d_{l, i}=\left|\begin{array}{cccc}
J_{0, i} & J_{1, i} & \cdots & J_{l, i} \\
J_{1, i} & J_{2, i} & \cdots & J_{l+1, i} \\
\vdots & \vdots & \ddots & \vdots \\
J_{l, i} & J_{l+1, i} & \cdots & J_{2 l, i}
\end{array}\right| \text {, }
\end{aligned}
$$




$$
K_{m l}^{i j}=\int_{-1}^{1} \int_{-1}^{1} \sum_{i, j=1}^{n} \frac{k^{i j}(x, \xi) p_{m}^{i \circ}(x) p_{l}^{j \circ}(\xi)}{m^{i}(x) m^{j}(\xi)} d x d \xi
$$

and coefficients $\gamma_{k}$ and $\psi_{k m}^{i}$ can be found from the spectral problem:

$$
\sum_{j=1}^{n} \sum_{l=2}^{\infty} K_{m l}^{i j} \psi_{k l}^{j}=\gamma_{k} \psi_{k m}^{i}, \quad i=1,2, \ldots, n \quad k, m=2,3,4, \ldots
$$

\section{Results and conclusions}

The contact-wear multiple plane problem for arbitrary system of rigid plane punches and elastic layer with nonuniform coating is formulated and solved. The efficient solution for one version of the problem is presented in analytical form. Solution constructing will be similar for all other versions.

Expressions for contact pressures under each punch have a structure in which the functions are associated with the coating nonuniformity are distinguished by separate factors. This fact allows one to carefully analyze the influence of contact characteristics on the stress-strain state of the foundation and positions of bodies. Moreover, such solution representation allows one to perform efficient calculations of multiple linear wear of layered foundations in cases when the coating elastic properties described by complex rapidly changing or even discontinuous functions.

Constructed analytical formulas allow to obtain asymptotic formulas for the main characteristics of contactwear problem.

\section{Acknowledgments}

The paper is financially supported by the Ministry of Science and Higher Education (State Registration Number AAAA-A17-117021310381-8) and, in part, by the Russian Foundation for Basic Research (under grant No. 18-51-05012-Arm_a).

\section{References}

Manzhirov, A.V., Kazakov, K.E. (2017) Contact problem with wear for a foundation with a surface nonuniform coating. Doklady Physics, 62, 7, pp 344-349. doi: 10.1134/S1028335817070035.

Manzhirov, A.V., Kazakov, K.E. (2018) Axisymmetric problem of fretting wear for a foundation with a nonuniform coating and rough punch. AIP Conference Proceedings, 1959, 070023. doi: 10.1063/1.5034698.

Kazakov, K.E., Kurdina, S.P., Manzhirov, A.V. (2017) Contact interaction between surface nonuniform bases and regular systems of rigid punches. Procedia IUTAM, 23, pp 201-209. doi: 10.1016/j.piutam.2017.06.021.

Kazakov, K.E. (2018) Contact between a regular system of punches and a layered foundation with consideration of complex surface shapes. AIP Conference Proceedings, 2053, 040041. doi: 10.1063/1.5084479.

Kazakov, K.E. (2019) On the indentation of a system of punches into a layered foundation. Engineering Letters, 27, $1, \mathrm{pp} 251-255$.

Alexandrov, V.M., Mkitaryan, S.M. (1979) Contact Problems for Bodies with Thin Coatings and Interlayers. Nauka, Moscow [in Russian].

Pronikov, A.S. (1957) Wear and Durability of Machines. Mashgiz, Moscow [in Russian].

Collins, J. (1993) Failure of Materials in Mechanical Design: Analysis, Prediction, Prevention. Wiley, New York.

Soldatenkov, I.A. (2010) Wear Contact Problem with Applications to Engineering Calculation of Wear. Fizmatkniga, Moscow [in Russian].

Alexandrov, V.M., Kovalenko, E.V. (1986) Problems in Mechanics of Continuous Media with Mixed Boundary Conditions. Nauka, Moscow [in Russian].

Vorovich, I.I., Alexandrov, V.M., Babeshko, V.A. (1974) Nonclassical Mixed Problems in the Theory of Elasticity. Nauka, Moscow [in Russian].

Archard, J.F. (1953) Contact and rubbing of flat surfaces. Journal of Applied Physics, 24, 8, pp 981-988. doi: $10.1063 / 1.1721448$.

Schalliamach, A. (1954) On the abrasion of rubber. Proceedings of the Physical Society. Section B, 67, 12, pp 883-891. doi: 10.1088/0370-1301/67/12/304.

Manzhirov, A.V. (2016) A mixed integral equation of mechanics and a generalized projection method of its solution. Doklady Physics 61, 10, pp 489-493. doi: 10.1134/S1028335816100025. 